\title{
Sinusoidal Supermolecular Structure of Band Textures in a Presheared Hydroxypropyl Cellulose Film
}

\author{
Jiandong DiNG, Jun FeNG, and Yuliang YANG \\ Department of Macromolecular Science and Laboratory of Molecular Engineering of Polymers, \\ Fudan University, Shanghai 200433, China
}

(Received July 4, 1995)

\begin{abstract}
A morphological study of hydroxypropyl cellulose (HPC) has been performed on solid films prepared by casting from a presheared liquid crystalline aqueous solution. Band textures were observed in a polarizing optical microscope. The sinusoidal supermolecular structure associated to the band texture was verified plausibly by comparison between the changes of experimental micrographs and theoretical birefringence patterns with the rotation of specimen. Some viewpoints in literature were commented. The pleated morphology was also confirmed in a scanning electron microscope.
\end{abstract}

KEY WORDS Liquid Crystalline Polymer / Band Texture / Supermolecular Structure / Hydroxypropyl Cellulose / Polarized Optical Microscopy /

Liquid crystalline polymer (LCP) exhibits distinct physical properties not to be expected in flexible polymers as well as in low molecular liquid crystals. One of interesting and puzzling phenomena is the band texture, which is common to all main chain LCP. The alternating dark and bright bands perpendicular to the preshearing direction was first observed in a polarizing microscope by Elliottt and Ambrose $^{1}$ from a solution cast film of poly $(\gamma$-benyl-L-glutamate) (PBLG), which was long before LCP became of wide interest since the appearance of Kevlar, a famous highmodulus fiber. The band texture was then observed in the very Kevlar ${ }^{2}$ and many other many chain LCPs. ${ }^{3-32}$ The bands are formed usually during shear relaxation, although occasionally, they can also be found during shear, ${ }^{29}$ or during elongation relaxation, ${ }^{27}$ or even without any noticeable macroscopic orientation. ${ }^{26}$ A peculiar side chain LCP with mesogenic units attached directly in a sideon mode can also exhibit band texture in the sheared specimen. ${ }^{33}$ Besides of interest in the light of the fundamental research, the band texture affects the mechanical properties of LCP materials very much ${ }^{28}$ and, therefore, has attracted much attention of scientists and engineers.

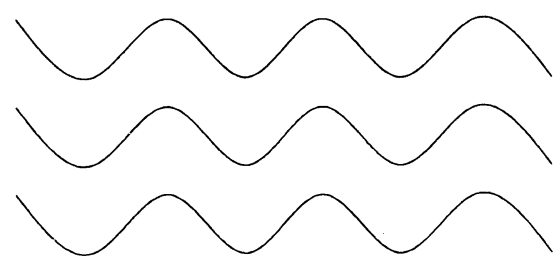

(a)

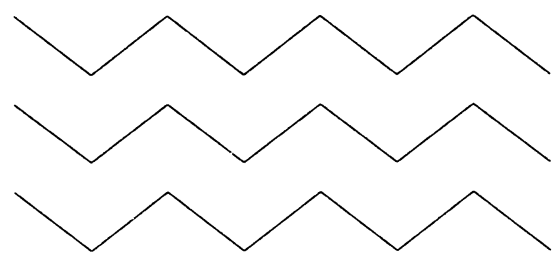

(b)

Figure 1. Schematic presentation of two ideal supermolecular structures of band textures. (a) sinusoidal model; (b) zigzag model. The preshearing direction is assumed to be horizontal. 
One of central questions in studies on band textures is, of course, the associated supermolecular structures. Even by polarized optical microscopy (POM), two typical periodic supermolecular structures of the band textures have been put forward in literature: one is the sinusoidal model as schematically presented in
Figure 1a; the other is the zigzag model as indicated in Figure 1b. The former is, to our knowledge, assumed first by Viney et al. ${ }^{7}$ in a copolyester of $p$-hydroxybenzoic acid, ethylene glycol and terephthalic acid (B-ET), while the latter, for instance, by Nishio et al. ${ }^{8}$ in hydroxypropyl cellulose (HPC). A question is,

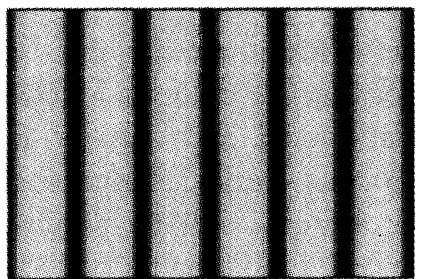

$0^{\circ}$

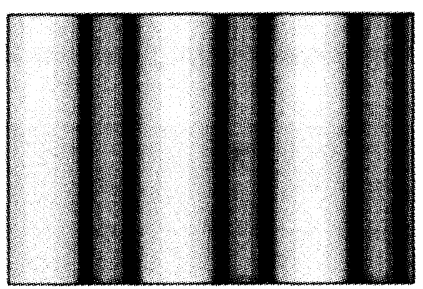

$10^{\circ}$

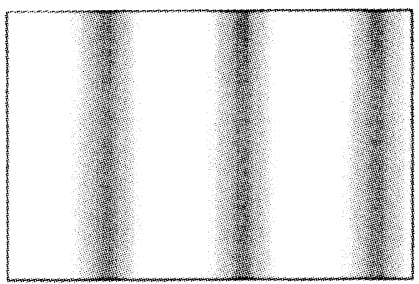

$30^{\circ}$

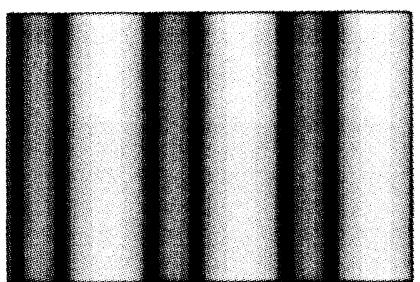

$80^{\circ}$

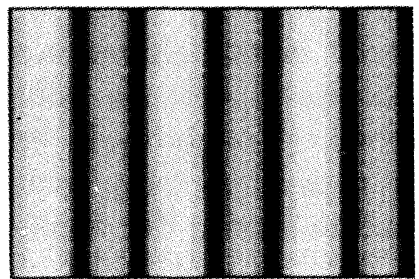

$5^{\circ}$

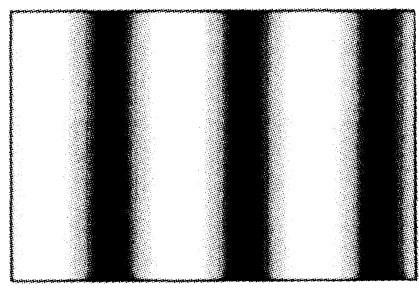

$20^{\circ}$

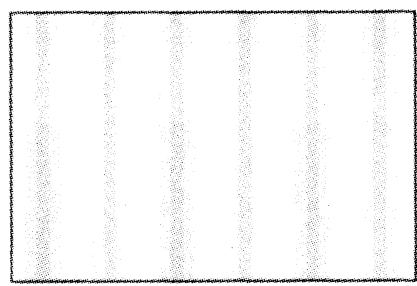

$45^{\circ}$

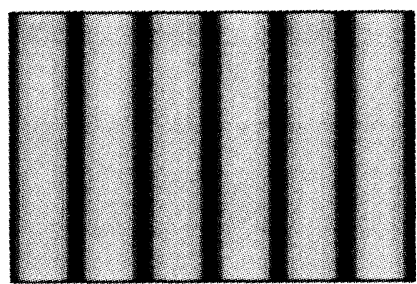

$90^{\circ}$

\section{preshearing direction}

Figure 2. Calculated birefringence patterns of the band texture with respect to the sinusoidal model under crossed polars. The director's maximum deviation angle from the preshearing direction, $\theta_{0}$, is assumed to be $20^{\circ}$. The noted angles indicate the polarizer orientation measured anticlockwise from the preshearing direction, which is along horizontal. 
however, open about the criterion to distinguish the two models by POM.

The criterion has been discovered by the authors. According to this, we have studied the film precipitated from a presheared nematic lyotropic solution of poly(1,4-phenylene terephthalamide) (PPTA) in sulfuric acid $\left(\mathrm{H}_{2} \mathrm{SO}_{4}\right)$ and the dried film of presheared HPC aqueous solution. Band textures of PPTA associated to both sinusoidal and zigzag models are observed. Hence, the two models are both reasonable. Corresponding results will be published in another paper. ${ }^{32}$ In the present paper, we would like to report the results about the HPC film. Different from Nishio et $a l .{ }^{8}$ we think that the band textures in our HPC films have the sinusoidal structure rather than the zigzag one.

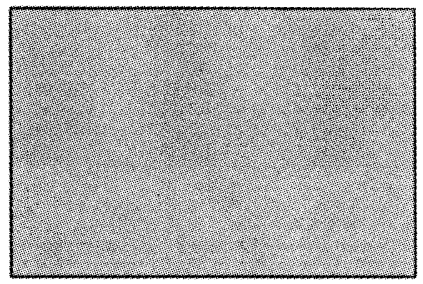

$0^{\circ}$

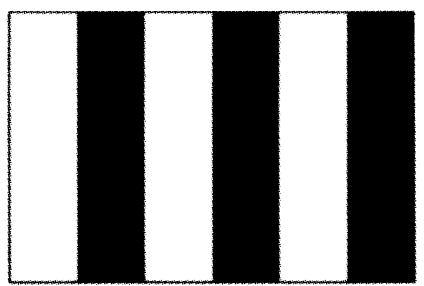

$20^{\circ}$

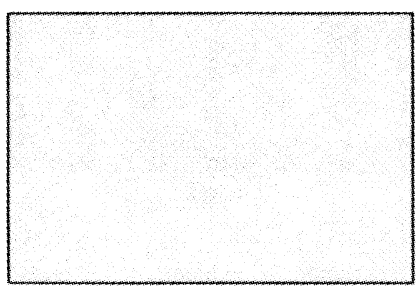

$45^{\circ}$
THEORETICAL

The birefringence patterns of anisotropic entities under orthoscopic polarizing observation can be calculated with the gridding method and displayed with the grey-pad method proposed by the authors. ${ }^{34-39}$ In this study, the supermolecular structures are assumed in two dimensions, and corresponding calculations are, therefore, easy to perform. The birefringence patterns associated to the sinusoidal model and zigzag model are shown in Figures 2 and 3, respectively. The calculation procedures will be published in ref 32 . The outputs indicate that both models can lead to periodic bands perpendicular to the preshearing direction in a polarizing microscope. When the specimen under observation is rotated with

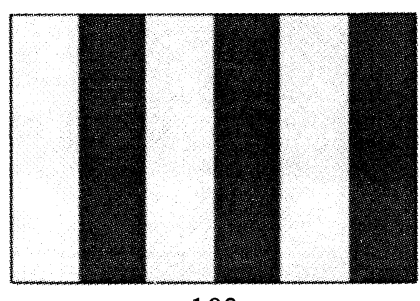

$10^{\circ}$
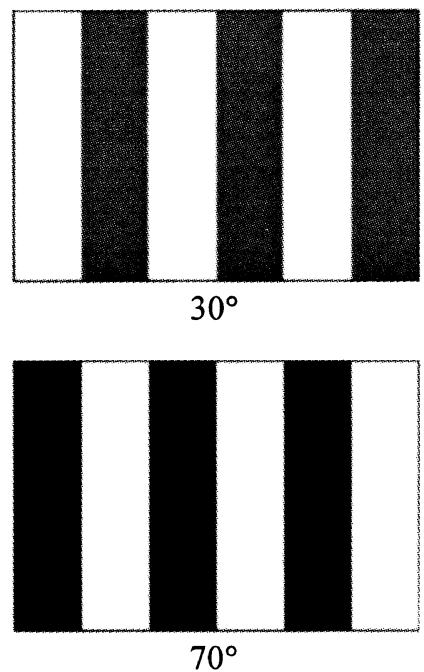

preshearing direction

Figure 3. The same as Figure 2 but with respect to the zigzag model. 
respect to the polarizer and analyser, the dark bands will be altered into bright ones and the bright bands into dark ones, when $\alpha$, the relative angle between preshearing direction and the polarizer is across $45^{\circ}$. (For convenience of comparison, it is better in actual photograph-taking to rotate the polars simultaneously whereas keeping the specimen fixed.) The contrast ratio shows strong dependence upon the angle $\alpha$, and is always large when $\alpha=\theta_{0}$, the maximum deviation angle between the local director and the preshearing direction. Such a phenomenon can be applied to measure $\theta_{0}$.

The most striking difference between the extinction behaviors of two supermolecular structures is that paired extinction bands can, depending upon the angle $\alpha$, occur for the sinusoidal model, while for the zigzag model, only the contrast ratio changes with $\alpha$. This point is just the key to distinguish these two supermolecular models. It seems worthy of further noting some other points. The paired bands for the sinusoidal model only occur when $0<\alpha<\theta_{0}$ (case I) if the angle $\alpha$ is, without loss of generality, limited within $0^{\circ}$ and $45^{\circ}$, and the band width $D$ with respect to $\alpha=0$ (case II) is only half of that with respect to $\theta_{0} \leq \alpha<45^{\circ}$ (case III) and is, hence, half of the band period $L$, although in both cases II and III, the bands are not divided into pairs. So we should be cautious to measure the period of band texture. For the zigzag model, the band width is kept constant in all of cases and the contrast ratio is even zero in the case II. No band texture is, therefore, noticeable in this case although the periodic orientation does exist in the specimen.

\section{EXPERIMENTAL}

HPC was supplied by TCI ( $M W 60000)$. The lyotropic liquid crystal was prepared by mixing HPC and water at room temperature followed by centrifugation to remove air bubbles. The solution $(46 \mathrm{wt} \%)$ was sheared between two glass slides in a simple self-built shear apparatus. The shear rate is about $1000 \mathrm{~s}^{-1}$. Immediately after shearing, the wet film was fast dried by a strong electric cold-wind-maker lasting for $20 \mathrm{~s}$. The dried film with depth about $20 \mu \mathrm{m}$ was observed in a Leitz polarizing optical microscope (POM). The source is the white light with orthoscopic incidence. The micrographs are captured by a CCD (charge coupled device) with $512 \times 512$ pixels and 256 relative intensity levels. The specimen was also observed in a Hitachi S-520 scanning electron microscope (SEM) after spurting $\mathrm{Au}$ on the film surface.

\section{RESULTS AND DISCUSSION}

The band textures are observed (Figure 4) and agree with the sinusoidal model. The paired

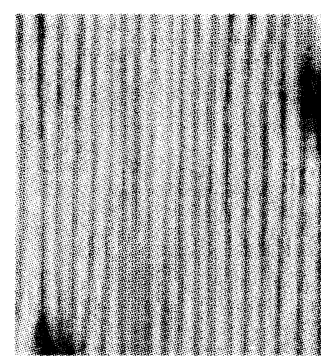

(a) $0^{\circ}$

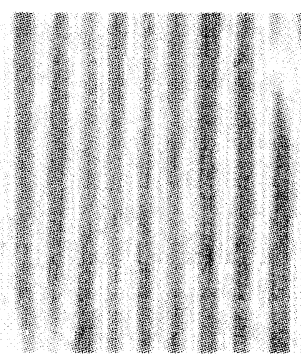

(c) $25^{\circ}$

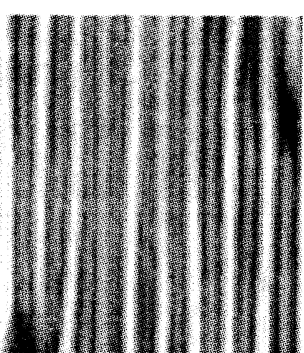

(b) $10^{\circ}$

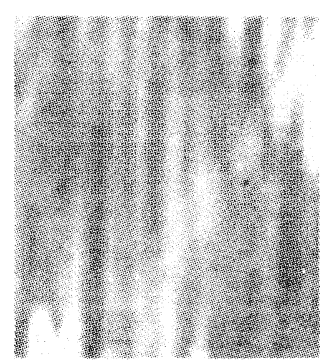

(d) $45^{\circ}$
$20 \mu \mathrm{m}$

\section{preshearing direction}

Figure 4. Polarizing micrographs of the presheared HPC film. (a) specimen in orthogonal position; (b) to (d) anticlockwise rotations of both polars away from this position by noted angles. The band period is about $4 \mu \mathrm{m}$. The maximum deviation angle of local directors from the preshearing direction shown by the arrow is near $25^{\circ}$. 
bands exist indeed when $0<\alpha<\theta_{0} \sim 25^{\circ}$ (Figure $4 b$ ). The band width and band number also change with the rotation of polars, which can be clearly seen by comparison between Figures 4a and 4c. Such a phenomenon is further confirmed with the polars fixed and the specimen rotated (Figures $5 \mathrm{a}$ and $5 \mathrm{~b}$ ). Especially, the quantitative measurements by CCD (Figures 5c and 5d) not only verifies the change of band width with the rotation of specimen, but also indicates the serpentine characteristic of the corresponding molecular orientations. As a consequence, our polarizing micrographs (Figures 4 and 5) show the orientational periodicity associated to the band texture and reveal that the corresponding supermolecular structure is sinusoidal rather than zigzag. Our POM observations are compatible with the recent SEM observations of HPC film by Patnaik et al. ${ }^{31}$ very well.

We would like to point out that although Nishio et al. proposed the zigzag model, ${ }^{8}$ their polarizing micrographs supported, in fact, the sinusoidal model, because the band number in the middle photograph (Figure 4 in ref 8 ) is twice of that in the top one, which is similar to the case of Figures $5 \mathrm{~b}$ and $5 \mathrm{a}$ in the present paper. A similar case occurred in ref 18 , where the zigzag model is assumed but their polarizing micrographs (Figure 1 in ref 18) indicate, in our opinion, the sinusoidal supermolecular structure, due to the change of band number. At this point, it seems necessary to mention that the difference between the sinusoidal and

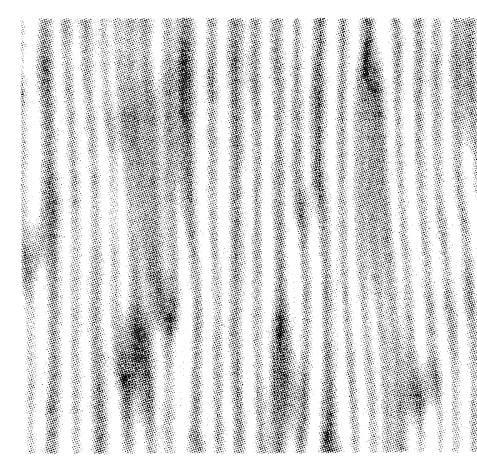

(a) $\alpha=0^{\circ}$

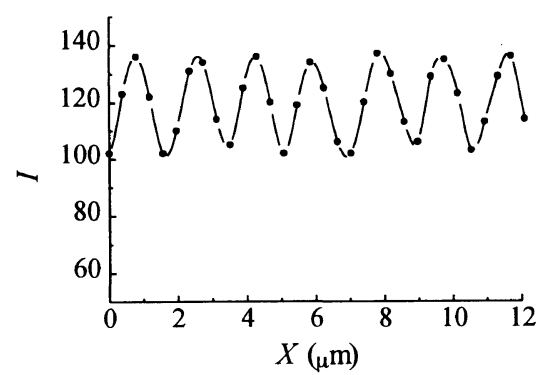

(c) $\alpha=0^{\circ}$

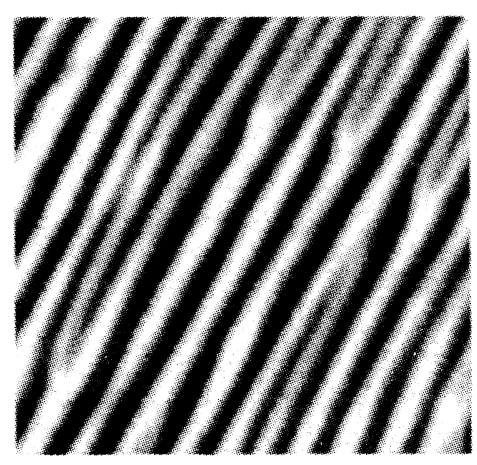

(b) $\alpha=30^{\circ}$

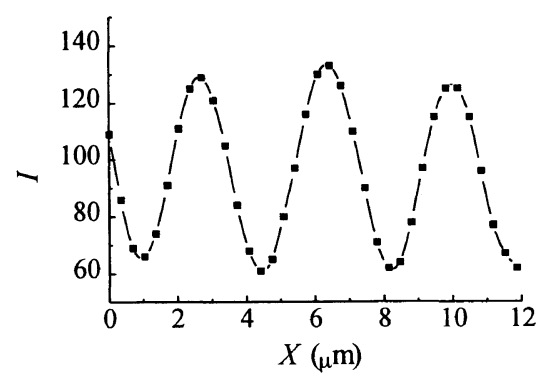

(d) $\alpha=30^{\circ}$

Figure 5. (a) and (b) Similar to Figures 4(a) and 4(c) but keeping the polars fixed and making the specimen rotated with the noted angles. The band period is near $4 \mu \mathrm{m}$. (c) and (d) Transmittance intensity $I$ as a function of position $X$ along the preshearing direction (vertical to the bands) for a small region in (a) and (b), respectively. The intensity is measured relatively by CCD with 256 intensity levels. 
zigzag models is not very discrete and sometimes, both model structures may be observed and converted to each other, according to our studies on the band textures of PPTA films. ${ }^{32}$ Considering the inevitable slight difference between the fine chemical strucures of the HPC samples and between the preparation conditions in this paper and in literature, our observation is not essentially contradictory to the previous studies. ${ }^{8,18} \mathrm{We}$ further notice that the supermolecular model proposed in ref 8 is a revised zigzag model with special kinks or boundaries between adjacent bands. But we still think that the sinusoidal model is more reasonable to explain the polarizing micrographs of our HPC films, considering the fact that the position of extinction bands can change with the rotation of specimen and the kinds do not always produce extinction.

As the dimensionality is concerned, the two-dimensional sinusoidal model of molecular orientation schematically shown in Figure 1a is something over simple. The pleated morphology can be observed by electron micrograph (Figure 6). The pleats were early observed by Nishio et al., ${ }^{8}$ and the contractional strain has been considered responsible for the out-of-plane component. This point is further conformed by us. It might be helpful to further indicate that the pleat period obtained by SEM ( $\sim 4 \mu \mathrm{m}$ according to Figure 6 ) is consistent with the band period obtained by POM (also $\sim 4 \mu \mathrm{m}$ according to Figures 4 and 5). This is very meaningful and implies that the orientation adjustment and the mass flow are coupled in the formation of the band textures. It seems that the backflow effect, which is, unfortunately, completely neglected in the previous unsuccessful explanations to the band textures, ${ }^{23,24}$ might be important. Further investigations with this effect taken into consideration is proposed by the authors.

It is necessary to indicate that actually, the internal director configuration might be beyond the two ideal models mentioned in this paper. The sinusoidal model refers, in the

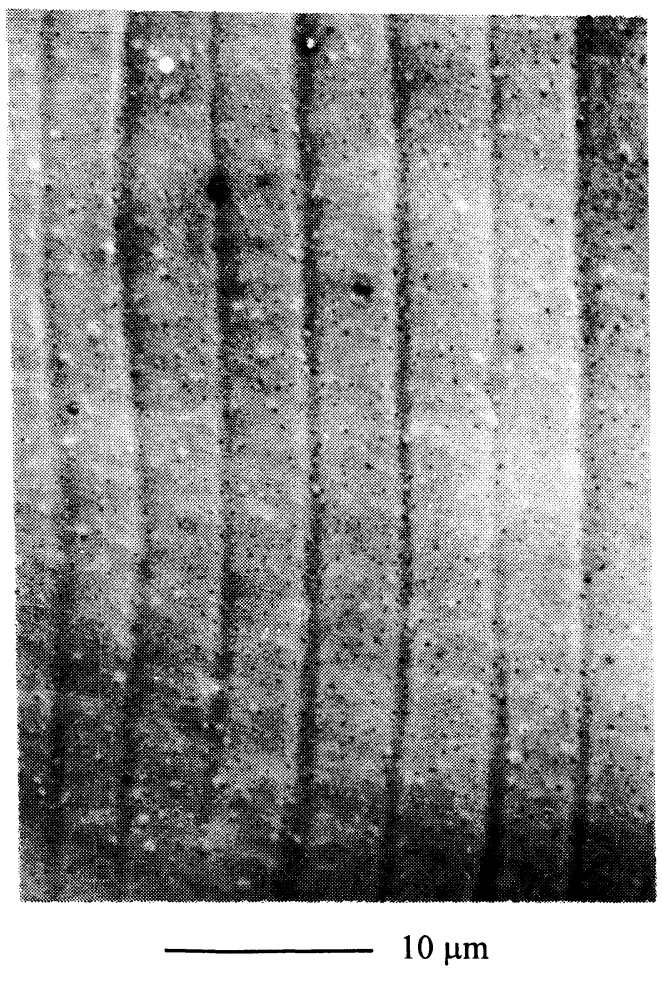

Figure 6. SEM micrograph of presheared HPC film.

general sense, to the serpentine director orientation. In some cases, the band texture may be finer and corresponding supermolecular structure might include helix and should be described in three dimensions. ${ }^{9.13,17,39}$ The effects of different supermolecular structures to the mechanical properties of the main chain LCP materials is far away from being resolved. The formation mechanism of the band texture has not been revealed up to now, although some hypotheses have been put forward and seem to be helpful. ${ }^{23,24}$ Hence, the band texture is still a quite open question at the present time.

Acknowledgments. This research was supported by NSF of China, Science and Technology Foundation of Shanghai, Chinese National Basic Research Project-Polymer Condensed State, and Foundation for Young Scientists in Fudan University No. CH21205. 


\section{REFERENCES}

1. A. Elliott and E. J. Ambrose, Discuss. Faraday Soc., 9, 246 (1950).

2. M. G. Dobb, D. J. Johnson, and B. P. Saville, J. Polym. Sci., Polym. Phys. Ed., 15, 2201 (1977).

3. S. C. Simmens and J. W. S. Hearle, J. Polym. Sci., Polym. Phys. Ed., 18, 871 (1980).

4. G. Kiss and R. S. Porter, Mol. Cryst. Liq. Cryst., 60, 267 (1980).

5. A. M. Donald, C. Viney, and A. H. Windle, Polymer, 24, 155 (1983).

6. R. J. Morgan, C. O. Pruneda, and W. Steele, J. Polym. Sci., Polym. Phys. Ed., 21, 1757 (1983).

7. C. Viney, A. M. Donald, and A. H. Windle, J. Mater. Sci., 18, 1136 (1983).

8. Y. Nishio, T. Yamane, and T. Takahashi, J. Polym. Sci., Polym. Phys. Ed., 23, 1053 (1985).

9. C. Viney, A. M. Donald, and A. H. Windle, Polymer, 26, 870 (1985).

10. P. Navard, J. Polym. Sci., Polym. Phys. Ed., 24, 435 (1986).

11. P. Navard and A. E. Zachariades, J. Polym. Sci., Polym. Phys. Ed., 25, 1089 (1987).

12. G. Marrucci, N. Grizzuti, and A. Buonaurio, Mol. Cryst. Liq. Cryst., 153, 263 (1987).

13. F. Fried and P. Sixou, Mol. Cryst Liq. Cryst., 158B, 163 (1988).

14. E. Marsano, L. Carpaneto, A. Ciferri, and Y. Wu, Liq. Cryst., 3, 1561 (1988).

15. E. Marsano, L. Carpaneto, and A. Ciferri, Mol. Cryst. Liq. Cryst., 158B, 267 (1988).

16. S. Chen, Y. Jin, R. Qian, and L. Cai, Makromol. Chem., 188, 2713 (1987).

17. T. M. Nicholson, Mol. Cryst Liq. Cryst., 177, 163 (1989).

18. S. Hu, M. Xu, B. Qian, X. Wang, and R. W. Lenz, J. Polym. Sci., Polym. Phys. Ed., 23, 2387 (1985).

19. S. Chen and R. Qian, Makromol. Chem., 191, 2475 (1990); Makromol. Chem. Macromol. Symp., 53, 345
(1992).

20. W. S. Putnam and C. Viney, Mol. Cryst. Liq. Cryst., 199, 189 (1991).

21. E. L. Thomas and B. A. Wood, Faraday Discuss. Chem. Soc., 79, 15 (1985).

22. B. Ernst and P. Navard, Macromolecules, 22, 1419 (1989).

23. J. T. Gleeson, R. G. Larson, D. W. Mead, G. Kiss, and P. E. Cladis, Liq. Cryst., 11, 341 (1992).

24. S. J. Picken, P. Moldenaers, S. Berghmans, and J. Mewis, Macromolecules, 25, 4759 (1992).

25. C. R. Fincher, Jr., Mol. Cryst. Liq. Cryst., 155, 559 (1988).

26. S. Chen, C. Du, Y. Jin, R. Qian, and Q. Zhou, Mol. Cryst. Liq. Cryst., 188, 197 (1990).

27. E. Peuvrel and P. Navard, Macromolecules, 24, 5683 (1991).

28. J. Wang and M. M. Labes, Macromolecules, 25, 5790 (1992).

29. N. X. Yan, M. M. Labes, S. G. Baek, and J. J. Madga, Macromolecules, 27, 2784 (1994).

30. J. Wang, S. Bhattacharya, and M. M. Labes, Macromolecules, 24, 4942 (1991).

31. S. S. Patnaik, T. J. Bunning, W. W. Adams, J. Wang, and M. M. Labes, Macromolecules, 28, 393 (1995).

32. J. Ding and Y. Yang, J. Polym. Sci., Polym. Phys. Ed., submitted.

33. G. Xu, J. Hou, S. Zhu, X. Yang, M. Xu, and Q. Zhou, Polymer, 35, 5441 (1994).

34. J. Ding and Y. Yang, Jpn. J. Appl. Phys., 31(9A), 2837 (1992)

35. J. Ding and Y. Yang, Funct. Polym., 6(1), 1 (1993) (in Chinese).

36. J. Ding, J. Zhu, and Y. Yang, Jpn. J. Appl. Phys., 33(A), 1400 (1994).

37. J. Ding and Y. Yang, Polym. Adv. Technol., 5, 603 (1994).

38. J. Ding, H. Zhang, J. Lu, and Y. Yang, Jpn. J. Appl. Phys., 34(4A), 1928 (1995).

39. J. Ding and Y. Yang, in preparation. 